\title{
Coronectomy: A Time to Act
}

\author{
Giuseppe Monaco \\ Clinical Professor of Oral Surgery \\ Department of Oral Surgery - Dental Clinic - University of Bologna, Italy
}

In 2004 Dr. Leon Assael wrote an editorial about Coronectomy after the publication of the article "Coronectomy: A Technique to Protect the Inferior Alveolar Nerve" of Pogrel, Lee and Muff in the Journal of Oral and Maxillofacial Surgery of 2004.

With the editorial "Coronectomy: A Time to Ponder or a Time to Act?" Dr. Assael wanted to stimulate the scientific discussion about this new technique.

Until 2004 a very few studies, based on the experience of some skilled surgeons have been published about Coronectomy. The results were good in terms of reduction of neurologic lesions but one of the most important points discussed by Dr. Assael was: "Empiricism does not rise to the level of scientific evidence of a prospective, randomized, double-blind clinical trial. In such a trial, at-risk patients would be randomly selected to undergo either coronectomy or odontectomy. The patient would not know which procedure had been performed (because of the subjective nature of reporting paraesthesia). The surgeon would not know the procedure to be performed until the random selection was made."

A study of this type was not easy to perform considering the ethical questions related. In Italy, the Ethics commitments usually do not permit the experimentation of a new technique on a patient if the tested procedure is not supported by consistent studies. Anyway, four prospective randomized, double blind studies have been published from 2005 to 2009 and in 2012, starting from these clinical trials, a systematic review and meta-analysis on Coronectomy has been published in the Journal of Dental Research. In the review authors concluded that "Coronectomy can protect inferior alveolar nerves in the extraction of third molars with high risk of nerve injury as compared to total removal, and that the risk ratios of post-operative infections were similar between the two surgical modalities." Moreover, they suggest that coronectomy could be used in clinical practice as an alternative to complete extractions in the case of third molars with a high risk of nerve injury but the surgical protocol should be improved.

In the years from 2009 to 2015 our research group (department of Oral Surgery, Dental Clinic University of Bologna - Italy) published some studies about Coronectomy. We proposed a wellstandardized surgical procedure in which the different steps were related to the type and position of third molar impaction. With this standardization, also less trained surgeons could obtain the same percentage of clinical success of more experienced surgeons.

A decade after Dr. Assael's editorial we can say that most of the points of criticism outlined from the clinicians unwilling to use the technique have been solved. The point that should be better investigated is the destiny of the pulp of these sectioned roots. We found that the risk of apical periodontitis of the sectioned roots is not significant, as shown in the 3 to 5 years follow-up of some studies. This aspect still requires deeper study. The pulp necrosis of the retained roots is less than $2 \%$ and this percentage is comparable to the risk of pulpitis after a crown restoration. In a recent histologic study, we found a vital pulp in a sectioned tooth removed after 18 months from coronectomy. The dental pulp is a tissue resistant to a mechanical trauma of the coronectomy procedure and the only request is the absence of bacterial contamination (caries of the crown) at the time of crown section. Recent studies published in the Journal of Dental Research confirmed this intrinsic capacity of self-repair of the vital pulp.

In conclusion, in the last six years, using our surgical protocol, we successfully performed more than 300 coronectomies without any failure or neurologic lesions. The technique requires a learning curve as every other surgical procedure and it could not be considered a remedy in case of difficult extraction but an alternative to complete third molar removal that must be decided before the first incision. 


\section{REFERENCES}

Pogrel MA, Lee JS, Muff DF: Coronectomy: A Technique to Protect the Inferior Alveolar Nerve. J Oral Maxillofac Surg 2004, 62: 1447-1452.

Assael LA: Coronectomy: A Time to Ponder or a Time to Act? J Oral Maxillofac Surg 2004, 62:1445-1446.

Renton T, Hankins M, Sproate C, McGurk M: A Randomized Controlled Clinical Trial to Compare the Incidence of Injury to the Inferior Alveolar Nerve as a Result of Coronectomy and Removal of Mandibular Third Molars. Br J Oral Maxillofac Surg 2005, 43: 7-12.

Hatano Y, Kurita K, Kuroiwa K, Yuasa H, Ariji E: Clinical Evaluation of Coronectomy (Intentional Partial Odontectomy) for Mandibular Third Molars Using Dental Computed Tomography: A Case Control Study. J Oral Maxillofac Surg 2009, 67:1806-1814.

Leung YY, Cheung LK: Safety of Coronectomy Versus Excision of Wisdom Teeth: A Randomized Controlled Clinical Trial. Oral Surg Oral Med Oral Pathol Oral Radiol Endod 2009, 108:821827.

Cilasun U, Yildirim T, Guzeldemir E, Pektas ZO: Coronectomy in Patients with High Risk of Inferior Alveolar Nerve Injury Diagnosed by Computed Tomography. J Oral Maxillofac Surg 2011, 69: 1557-1561.

Long H, Zhou Y, Liao L, Pyakurel U, Wang Y, and Lai W: Coronectomy vs. Total Removal for Third Molar Extraction: A Systematic Review. J Dent Res 2012, 91(7):659-665.

Monaco G, de Santis G, Gatto MR, Corinaldesi G, Marchetti C. Coronectomy: A Surgical Option for Impacted Third Molars in Close Proximity to the Inferior Alveolar Nerve. J Am Dent Assoc 2012, 143: 363-369.

Monaco G, de Santis G, Diazzi M, Marchetti C: Coronectomy of Mandibular Third Molars. Inside Dentistry February 2013.

Costabile E, Vignudelli E, Kellezi A, Monaco G, Marchetti C: Good Neurological Results with Coronectomy in Third Molar Surgery: Case Series Study. Rivista Italiana di Chirurgia MaxilloFacciale - Maggio 2013.

Monaco G, de Santis G, Marchetti C: Coronectomia: Analisi della Letteratura ed Illustrazione del Protocollo Chirurgico. Dental Cadmos 2013, 81:437-445.

Monaco G., Vignudelli E., Diazzi M., Marchetti C., Corinaldesi G: Coronectomy of Mandibular Third Molars: a Clinical Protocol to Avoid Inferior Alveolar Nerve Injury. J Cranio Maxillofac Surg, Oct 43:(8)1694-99, 2015.

Monaco G., De Santis G., Gatto MRA., Vignudelli E., Marchetti C: What Are the Types and Frequencies of Complications Associated with Mandibular Third Molar Coronectomy? A Follow-up Study. J Oral Maxillofac Surg, July 73(7)1246-53 2015.

Vignudelli E., Monaco G., Mazzoni A., Marchetti C: Root Fragment Vitality after Coronectomy: Histological Evidence in a Case. J Oral Maxillofac Surg, Nov 73(11)2093-5, 2015 